\title{
Dégradation microbienne dans le rumen de la tige de blé à différents stades de développement, observée au microscope électronique à balayage
}

P. BARRY, Elisabeth GRENET

Unité de l'Ingestion, I.N.R.A., Theix, 63122 Ceyrat, France.

Summary. Rumen microbial digestion of wheat stem at different stages of growth has been observed by light microscope and scanning electron microscope.

Le degré de lignification à partir duquel un tissu ne peut pas être dégradé dans le rumen étant mal connu, nous avons, à l'aide des microscopes photonique (MP) et à balayage (MEB), suivi l'évolution de la lignification des tissus de la tige de blé et leur dégradation dans le rumen.

Matériel et méthodes. Des tiges de blé (Triticum vulgaris) ont été récoltées, à deux semaines d'intervalle, du stade montaison jusqu'à la maturité du grain. Des fragments de $0,5 \mathrm{~cm}$ de long ont été prélevés à mi-hauteur de l'entre-nœud supérieur et pour partie introduits dans des sachets de nylon, à raison de 6 par sachet, puis placés pendant $8,24,48$ et $72 \mathrm{~h}$ dans le rumen d'une vache fistulisée, alimentée avec du foin de prairie naturelle; à leur sortie du rumen ils ont été préparés pour l'observation au MEB, ainsi que 4 échantillons témoins, (Grenet et Barry, 1988). Des coupes à main levée ont été réalisées sur des fragments supplémentaires avant passage dans le rumen afin de mettre en évidence la présence des composés phénoliques (précurseurs de la lignine) dans les parois par fluorescence dans I'UV (Harris et Hartley, 1976), et de la lignine par la coloration au phloroglucinol-chlorhydrique. La digestibilité de l'entre-nœud supérieur a été déterminée par la méthode à la pepsine-cellulase (Aufrere, 1982).

Résultats et discussion. A la montaison, les tissus, constitués dans leur quasi totalité de petites cellules à parois minces, sont très digestibles (tabl. 1); seul le xylème est lignifié et reste intact. A l' épiaison (photo 1), la taille des cellules augmente et leurs parois s'épaississent mais, bien que le sclérenchyme et le parenchyme émettent tous deux une intense fluorescence dans I'UV (tabl. 1), seul le sclérenchyme, par ailleurs coloré par le phloroglucinol, n'est pas dégradé après $72 \mathrm{~h}$ de séjour dans le rumen (photo $1 \mathrm{a}$ ). Au fur et à mesure que la plante vieillit les parois du parenchyme se lignifient progressivement de l'extérieur vers l'intérieur de la tige, comme l'atteste leur coloration par le phloroglucinol; simultanément elles deviennent indégradables dans le rumen. A la maturité du grain (photo 2), seule la frange du parenchyme qui entoure la lacune médullaire est dégradée (photo $2 a$ ) et la dégradation de l'entre-nœud diminue fortement (tabl. 1). Au début de la lignification, la dégradation des polyosides des parois du parenchyme est freinée par la présence de précurseurs de la lignine; le parenchyme qui en est dépourvu au stade montaison est dégradé dès $8 \mathrm{~h}$ de séjour, alors qu'au stade épiaison où on décèle leur présence, sa dégradation demande $72 \mathrm{~h}$. 

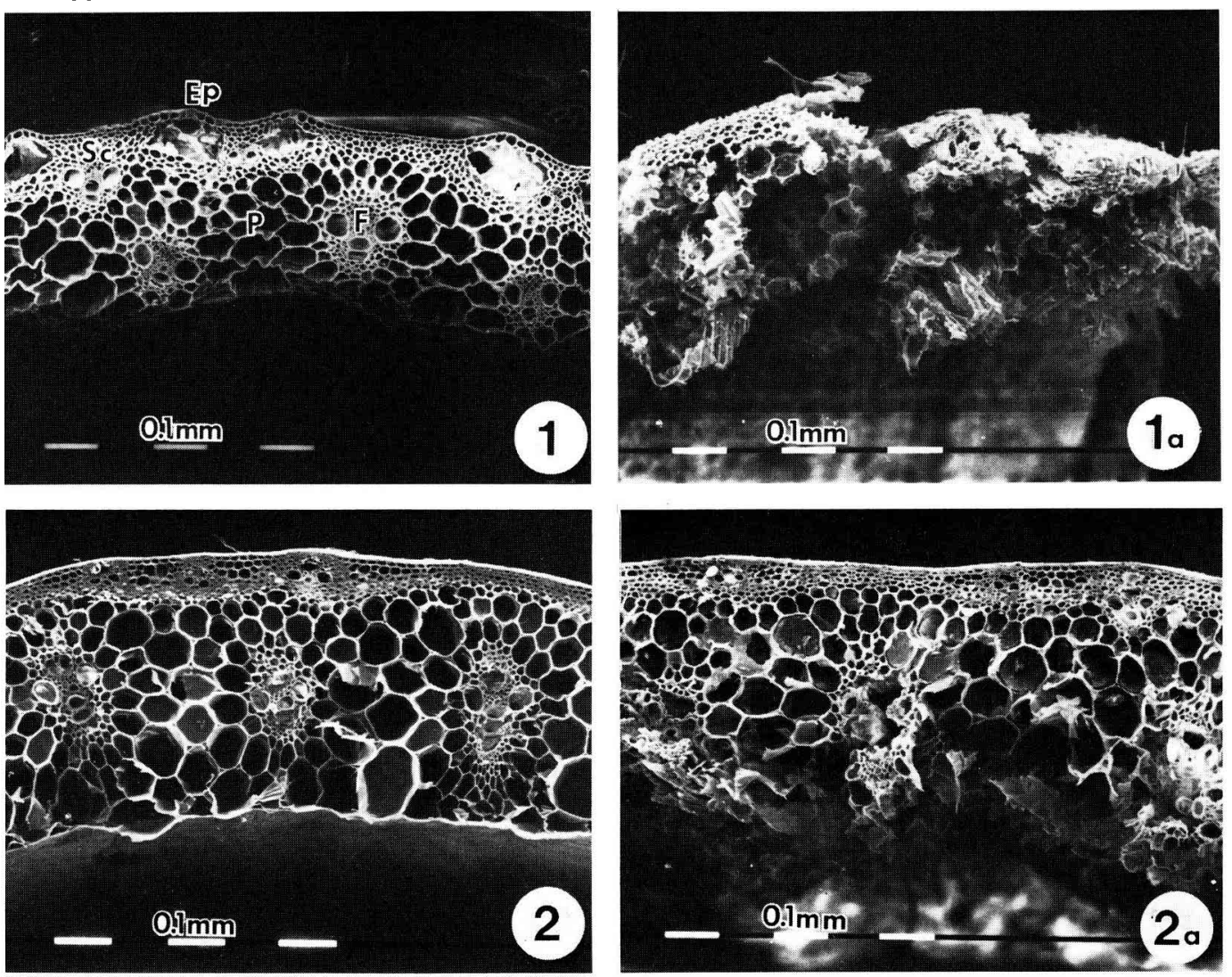

Photo 1. - Stade épiaison, les différents tissus se différencient: (de haut en bas), l'épiderme (Ep), le sclérenchyme (Sc), le parenchyme (P), les faisceaux libéro-ligneux (F). Photo 1a. - Stade épiaison, $72 \mathrm{~h}$ de séjour dans le rumen. les faisceaux libéro-ligneux se sont détachés et résistent à la dégradation, de même que les fragments du sclérenchyme en début de lignification. Photo 2. - Stade maturité du grain. Photo 2a. - Stade maturité du grain. $72 \mathrm{~h}$ de séjour dans le rumen, seul le parenchyme le plus interne non lignifié est dégradé par les microorganismes.

TABL. 1. - Autofluorescence (F), et réaction au phlorog/ucinol-chlorhydrique (PC) ( + : positive, - : non détectable) et dégradation dans le rumen (D), ( $8 \mathrm{~h}$ et $72 \mathrm{~h}$ de séjour), ( $0:$ nulle, $0^{+}:$faible, 1 : totale).

\begin{tabular}{|c|c|c|c|c|c|c|c|c|c|}
\hline \multirow[t]{3}{*}{ Stades } & \multicolumn{4}{|c|}{ Sclérenchyme } & \multicolumn{4}{|c|}{ Parenchyme } & \multirow{3}{*}{$\begin{array}{c}\text { Entre-noud } \\
\text { Digestibilité } \\
\text { pepsine- } \\
\text { cellulase }\end{array}$} \\
\hline & \multirow[t]{2}{*}{$\mathrm{F}$} & \multirow[t]{2}{*}{$\mathrm{PC}$} & \multicolumn{2}{|c|}{$\mathrm{D}$} & \multirow[t]{2}{*}{$\mathrm{F}$} & \multirow[t]{2}{*}{$\mathrm{PC}$} & \multicolumn{2}{|c|}{$\mathrm{D}$} & \\
\hline & & & $8 \mathrm{~h}$ & $72 \mathrm{~h}$ & & & $8 \mathrm{~h}$ & $72 \mathrm{~h}$ & \\
\hline Montaison & - & - & 1 & 1 & - & - & 1 & 1 & 67 \\
\hline Epiaison & + & + & 0 & $0^{+}$ & + & - & 0 & 1 & 56 \\
\hline Maturité du grain & + & + & 0 & 0 & + & + & 0 & $0^{+}$ & 20 \\
\hline
\end{tabular}

Aufrère J., 1982. Ann. Zootech., 31, 111-130.

Grenet E., Barry P., 1988. Anim. Feed Sci. Technol., 19, 25-31.

Harris P. J., Hartley R. D., 1976. Nature, 259, 508-510. 